\title{
A luminosity monitor for the Mark II detector at the SLC
}

\author{
J. Hylen ${ }^{1}$ and J.A.J. Matthews ${ }^{2}$ \\ The Johns Hopkins Unicersity, Baltimore, MD, USA
}

\author{
G. Bonvicini ${ }^{3}$, J. Chapman, R. Frey ${ }^{4}$, S.J. Hong, E. Gero, M. Petradza ${ }^{5}$, R. Thun \\ and $\mathrm{H}$. Veltman ${ }^{6}$ \\ University of Michigan, Ann Arbor, MI, USA
}

Received 21 February 1992

We describe the design and performance of the Mark II small angle monitor (SAM) used for measurements of Bhabha scattering at the SLC. We also present results of SLC luminosity measurements made with SAM for the first determination of Z boson resonance parameters in $\mathrm{e}^{+} \mathrm{e}^{-}$annihilation.

\section{Introduction}

Detection of Bhabha events, $\mathrm{e}^{+} \mathrm{e}^{-} \rightarrow \mathrm{e}^{+} \mathrm{e}^{-}$, at wide angles to the beam axis has traditionally been used for precision luminosity measurements at $\mathrm{e}^{+} \mathrm{e}^{-}$colliders. However, at the new higher energy colliders, SLC and LEP, the wide angle Bhabha rate is too low, and is in fact dominated by the weak interaction that one is trying to study. We are thus forced to use Bhabha events at angles close to the beam line for the luminosity measurement. One then has to control added systematic effects due to the steeply falling spectrum ( $d \sigma / d \theta \propto \theta^{-3}$, where $\theta$ is the angle from the beam axis; and higher backgrounds near the beam line.

The small angle monitor (SAM) was designed for use with the Mark II detector at the SLC [1]. The front of the SAM (fig. 1) contains tracking chambers to measure the direction of the final state $\mathrm{e}^{ \pm}$. The rear portion is a shower counter, where the $\mathrm{e}^{ \pm}$(and photon for $\mathrm{e}^{+} \mathrm{e}^{-} \rightarrow \mathrm{e}^{+} \mathrm{e}^{-} \gamma$ events) are detected by their large energy depositions. The major design goals, dictated by previously mentioned systematic considerations, were good resolution in $\theta$ in the tracking chambers and fine grained calorimetry for background suppression.

\footnotetext{
1 Present address: Fermilab, Batavia, USA.

2 Present address: University of New Mexico, Albuquerque, USA.

3 Present address: CERN, Geneva, Switzerland.

${ }^{4}$ Present address: University of Oregon, Eugene, USA.

5 Present address: SLAC, Stanford, USA.

6 Present address: DESY, Hamburg, Germany.
}

This paper includes a description of the SAM detector, its performance during the April to October 1989 SLC running, and the analysis used to determine the integrated luminosity for the run. Data were taken at ten center-of-mass energies, ranging from 90 to 93 GeV. We detected 839 Bhabha events in the SAM and $455 \mathrm{Z}^{0}$ hadronic decays in the Mark II.

\section{Detector description}

The SAM modules, located $1.4 \mathrm{~m}$ from the interaction point at each end of the Mark II detector, cover the angular range $\theta=50$ to $165 \mathrm{mrad}$ from the beam axis. The tracking section, located in the front part of

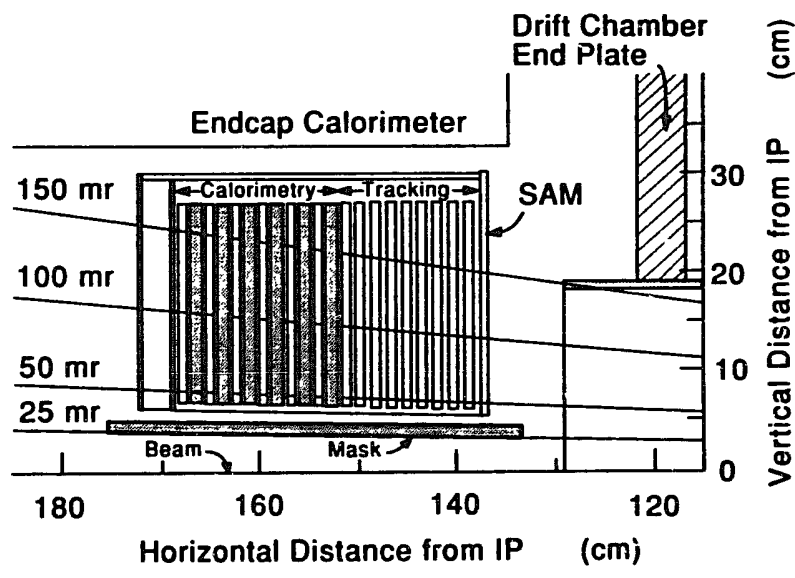

Fig. 1. Cutaway view of SAM. 
each module, consists of nine layers of drift tubes. The shower counte $\mathrm{cm}$ of lead followed by sampling proportional tubes. Every drift and shower counter layer contains sixty $0.95 \times 0.95 \mathrm{~cm}$ square aluminum tubes with a wall thickness of $0.025 \mathrm{~cm}$. A $38 \mu \mathrm{m}$ diameter gold-plated tungsten sense wire is centered within each tube. Layers are arranged in zriplets at zero and $\pm 30^{\circ}$ with respect to the horizontal direction to provide stereo reconstruction. Each SAM consists of two identical modules, as shown in fig. 2 , which could be installed and removed without disturbing the beam pipe. When fitted together, the modules provide complete azimuthal coverage. Table 1 gives a more detailed description of the SAM detector. The drift and shower tubes were operated at $1800 \mathrm{~V}$ and $1700 \mathrm{~V}$, respectively, using a gas mixture consisting of $89 \%$ argon, $10 \%$ carbon dioxide, and $1 \%$ methane.

The signals from the wires in the SAM tracking system were discriminated using LeCroy Research Systems LD604 chips whose threshold was set to $0.5 \mathrm{mV}$. The time delays of these discriminated signals, corresponding to the drift times in the tracking tubes, were stored in SLAC TAC CAMAC modules [2] and digitized using SLAC BADCs [3]. The signals from the wires in the shower counter were amplified by two-stage hybrid chips with a response of approximately $3 \mathrm{~V}$ output for $100 \mathrm{pC}$ input. The amplitudes of the pulses were stored in SLAC SHAM modules [2] and digitized using SLAC BADCs. The LD604 and amplifier cards were located in crates with custom backplanes, which also allowed the distribution of calibration pulses to the inputs of the electronics chain.

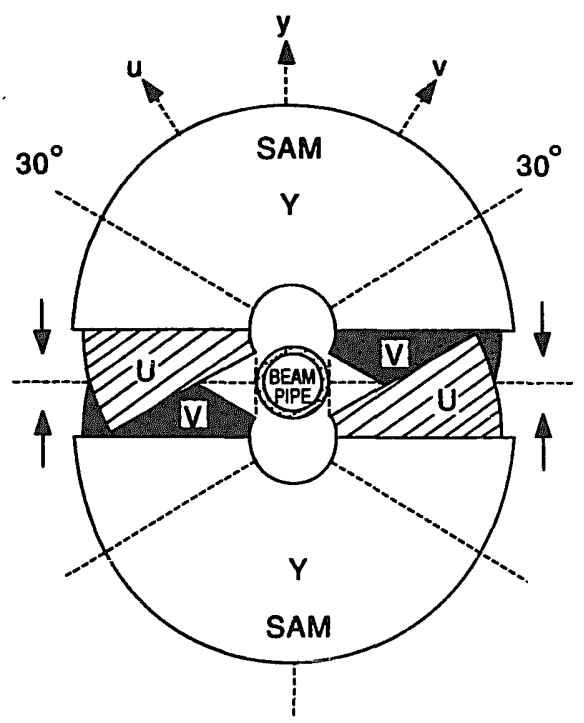

Fig. 2. Front view of SAM being inserted around the beampipe.
Table 1

Positions and orientations of detector layers. The outer radius is $0.274 \mathrm{~m}$ for all layers. Above values are for the south SAM; change signs for all $Z$ and angle values for the north SAM

\begin{tabular}{clllc}
\hline Layer & Function & $Z[\mathrm{~m}]$ & $\begin{array}{l}\text { Inner radius } \\
{[\mathrm{m}]}\end{array}$ & $\begin{array}{l}\text { Stereo angle } \\
\text { [degrees] }\end{array}$ \\
\hline 1 & Tracking & 1.4018 & 0.070 & 0 \\
2 & Tracking & 1.4176 & 0.071 & 30 \\
3 & Tracking & 1.4335 & 0.071 & -30 \\
4 & Tracking & 1.4494 & 0.072 & 0 \\
5 & Tracking & 1.4653 & 0.073 & 30 \\
6 & Tracking & 1.4811 & 0.073 & -30 \\
7 & Tracking & 1.4970 & 0.074 & 0 \\
8 & Tracking & 1.5129 & 0.074 & 30 \\
9 & Tracking & 1.5288 & 0.075 & -30 \\
10 & Shower & 1.5573 & 0.076 & 0 \\
11 & Shower & 1.5859 & 0.076 & 30 \\
12 & Shower & 1.6145 & 0.077 & -30 \\
13 & Shower & 1.6431 & 0.078 & 0 \\
14 & Shower & 1.6716 & 0.079 & 30 \\
15 & Shower & 1.7002 & 0.080 & -30 \\
\hline
\end{tabular}

The SAM trigger used analog sums of the signals from the wires in the calorimeter layers. To provide redundancy in the trigger, each SAM calorimeter is segmented into back and front halves, each with a trigger energy threshold of $4 \mathrm{GeV}$. Either half of the north module above threshold in coincidence with either half of the south module caused a trigger. For most of the running, a total energy deposit of $7 \mathrm{GeV}$ in either the north or south module alone would also cause a trigger. This threshold is well below the 18 $\mathrm{GeV}$ per module minimum required for off-line Bhabha event selection. The trigger inefficiency is negligible within the fiducial region of $60<\theta<160 \mathrm{mrad}$.

Several other signals were digitized and read out on triggered events for diagnostic purposes. These included the trigger sum pulse heights, latches of trigger patterns, and the timing of SAM module gating signals. Counts of trigger hit patterns from non-triggered events were also recorded.

\section{Detector operation}

The SAM was in operation for more than a year, with no major hardware problems. One of the gate signals for the TAC units did fail during a few hours of data taking. However, no Bhabha events occured during that time.

On average, about 20 of 1080 TAC tracking channels and 1 or 2 (maximum 5) of 720 SHAM calorimeter channels failed calibration, and were not usable. The effect of the worst case of five failing calorimeter 
channels was evaluated using a Bhabha Monte Carlo, in which SAM showers were simulated with EGS [4]. The result was a $0.3 \%$ increase in the fiducial cross section, a negligible effect compared to statistical errors.

\section{Event reconstruction}

Two sets of algorithms for SAM track and shower reconstruction were developed and compared [5]. For the shower reconstruction, the first algorithm begins by taking a wire whose pulse height is above a preselected threshold of $50 \mathrm{MeV}$. It then iteratively adds wires to the cluster which are above threshold and are adjacent to the clustered wires or separated by only a single below-threshold wire. The second algorithm picks the wire with the highest pulse height and adds the two or four adjacent wires to form a cluster. It then starts a new cluster with the highest remaining wire, and so on. Two adjacent wires are used for the first and last calorimeter layers; four are used for the middle layers. Clusters from the different layers are then assembled into reconstructed showers by fitting the centers-ofgravity of the clusters to a straight line originating from the interaction point. Longitudinal shower leakage corrections are made based on the shower profile in the last two layers. This correction averages about $10 \%$ for $46 \mathrm{GeV}$ showers. Both algorithms gave similar results; for this analysis we used the two/four adjacent wire algorithm.

Offsets of the detector relative to the colliding beams were derived from reconstructed Bhabha events. In the limit that Bhabha events are exactly collinear, the $X$ or $Y$ position of the interaction point (IP) is just the average of the $X$ or $Y$ coordinates of the showers

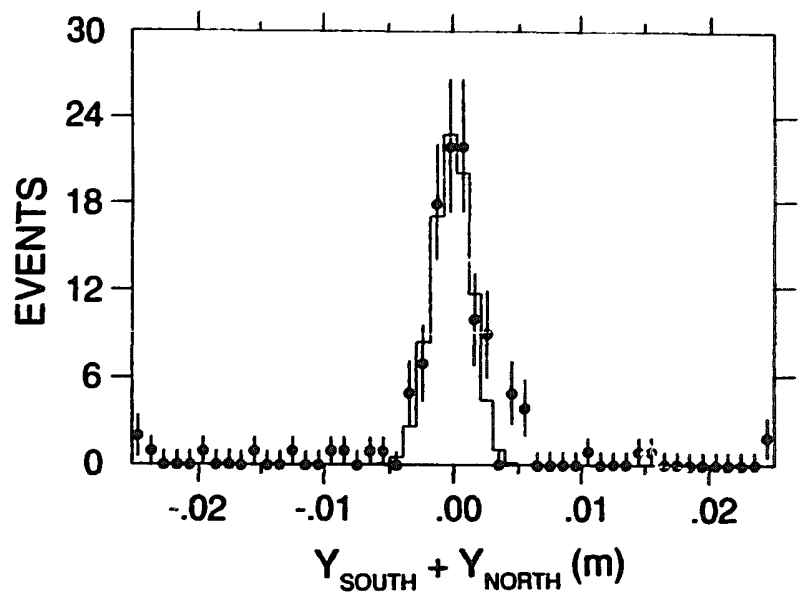

Fig. 3. Sum of $Y_{S}$ (shower $Y$ position in south SAM) and $Y_{N}$ (shower $Y$ position in north SAM) for Bhabha events for one set of runs. The histogram is a Gaussian fit.

in the north and south modules. In practice, a sample of a few dozen events gave a very good measurement of the IP position with respect to the SAM as indicated in fig. 3.

Fig. 4 shows the reconstructed $X$ and $Y$ position of the IP using Bhabha events in the SAM and hadronic $Z^{0}$ decay events in the Mark II central drift chamber. A survey of the relative positions of the detectors found them to be essentially aligned in $X$, and the central drift chamber to be $0.5 \mathrm{~mm}$ higher than the SAM in $Y$. The excellent agreement between the reconstruction offsets and survey offsets indicates a good understanding of their relative alignment and their respective track reconstruction.

A Gaussian fit to the sum of north and south shower $Y$ positions (shown in fig. 3) has a standard deviation of $1.5 \mathrm{~mm}$, implying a shower $Y$ position
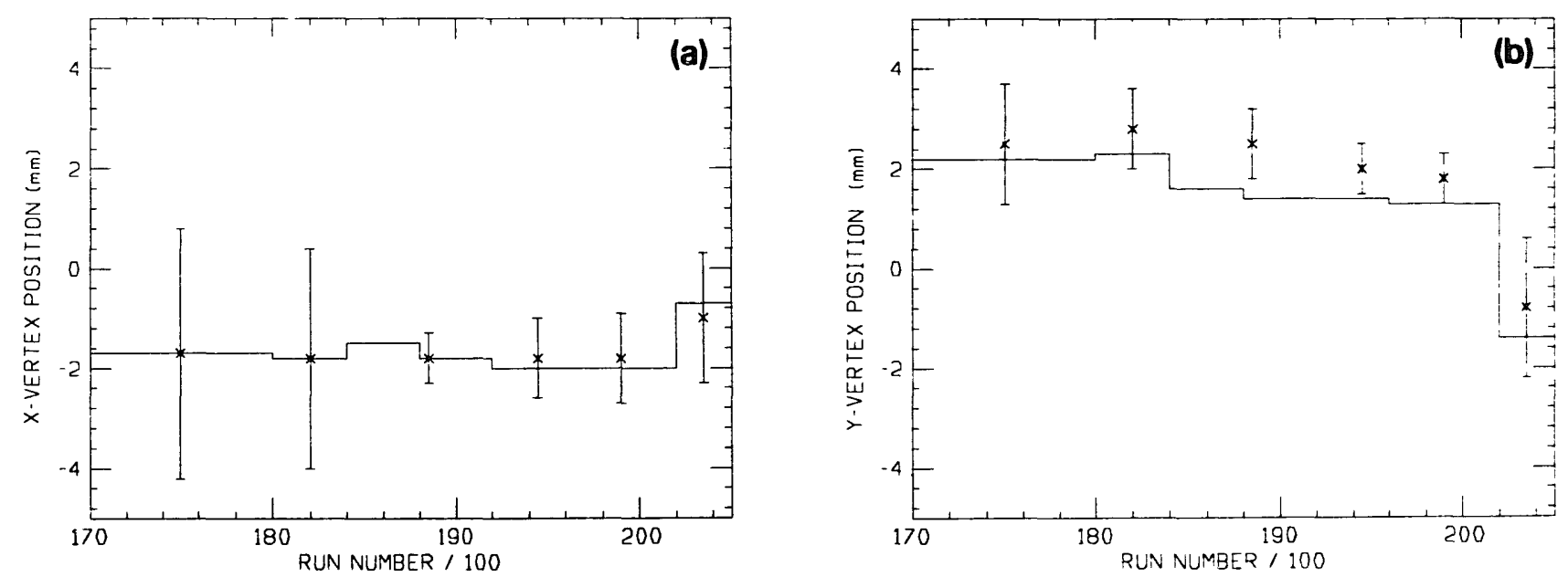

Fig. 4. (a) The reconstructed $X$ position of the IP from SAM data (crosses) and from the Mark II Central Drift Chamber data (histogram). The data are roughly divided into periods of differing beam alignment. (b) Same as (a) but for $Y$ position. 


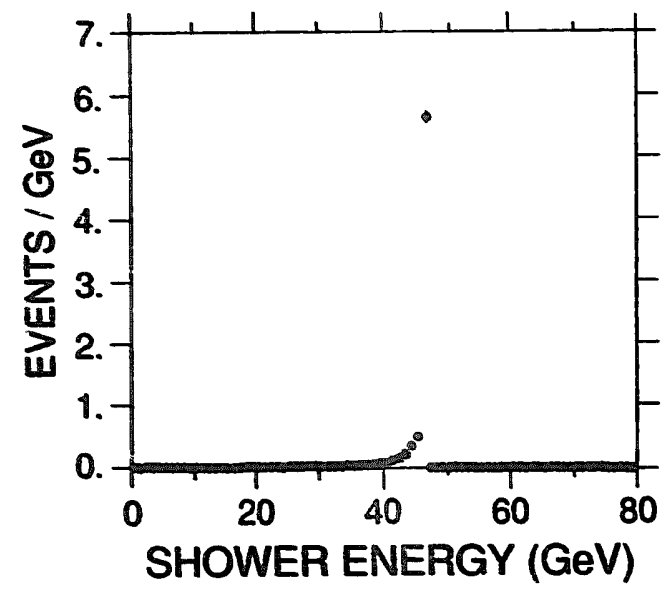

Fig. 5. Energy of $\mathrm{e}^{+}, \mathrm{e}^{-}$or $\boldsymbol{\gamma}$ in the SAM Inclusive fiducial region from a Bhabha Monte Carlo, neglecting detector resolution effects.

resolution in each module of about $1 \mathrm{~mm}$ (or less, since we have not unfolded the intrinsic Bhabha acollinearity from the width of the distribution). The resolution in the $X$ direction is about twice that, because of the $30^{\circ}$ stereo wire pattern. The $\theta$ resolution of the shower measurements is thus $\phi$ dependent, but of the order of $1 \mathrm{mrad}$.

The expected Bhabha energy distribution, without detector resolution effects, is shown in fig. 5. The distribution is very sharpiy peaked at the beam energy, with only a small tail due to photon radiation. The energy distribution expected when shower fluctuations

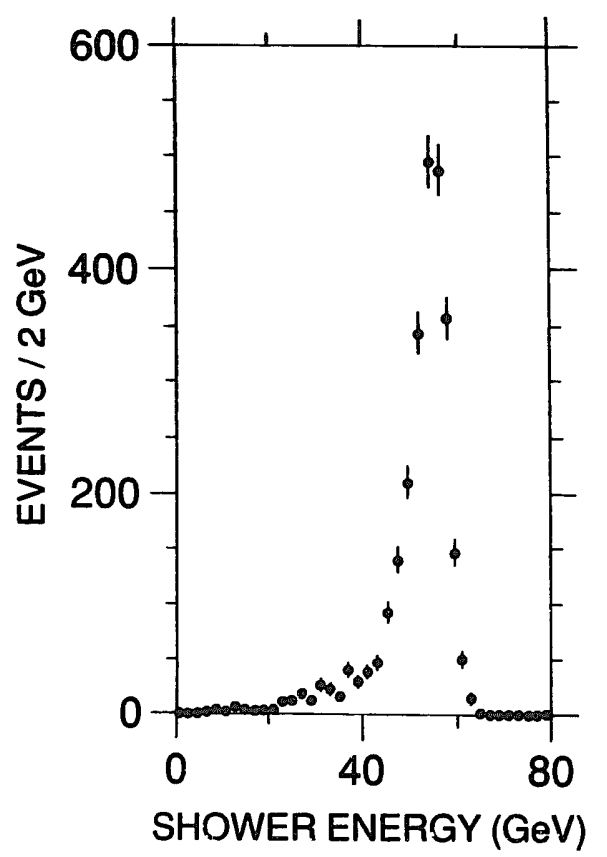

Fig. 6. Energy of Bhabha showers in the SAM Precise fiducial region predicted from EGS Monte Carlo.

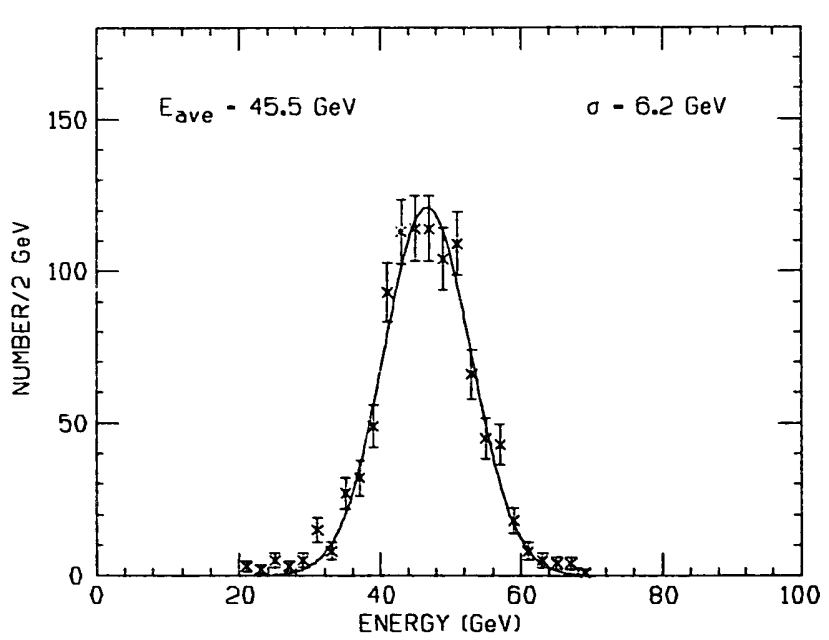

Fig. 7. Reconstructed shower energy in back-to-back SAM events for $60<\theta<115 \mathrm{mrad}$. The energy scale is normalized to the beam energy. Gas gain, saturation, and longitudinal leakage corrections are included.

are included (that is, the resolution predicted by an EGS model) is shown in fig. 6.

The energy resolution of the SAM is evaluated in two angular regions: the "clean" region $60<\theta<115$ mrad, and the "preshower" region $115<\theta<160 \mathrm{mrad}$ where several radiation lengths of material in front of the SAM degrade resolution. A plot of the shower energy in the "clean" region (fig. 7) shows a Gaussianshaped peak with practically no tail near the cut of $E_{\text {shower }}>0.4 E_{\text {beam }}$. A fit to the peak gives $(14 \pm 2) \%$ for the energy resolution. Both the north and south modules have the same resolution, and after corrections of $5 \%$ to $10 \%$ for temperature differences both give the same peak positions. Fig. 8 shows the effect on reconstructed-energy resolution of the extra material in front of the SAM in the large-angle "preshower" region. This region comprises $15 \%$ of the SAM Bhabha cross section, and its inclusion slightly lessens the total luminosity error because of the increased data sample. With higher statistics running, this region would be excluded to minimize systematic errors.

\section{Saturation}

The energy resolution attaned in the "clean" region, although certainly sufficient to cleanly tag Bhabha events, is worse than expected from the simple EGS simulation. Much of this discrepancy is caused by a saturation effect, which is attributed to local space charge produced when large energy depositions occur on small regions of a wire. The size of this effect can be reduced by running with lower voltage. However, we kept the voltage sufficiently high to observe minimum ionizing signals, such as produced by background 


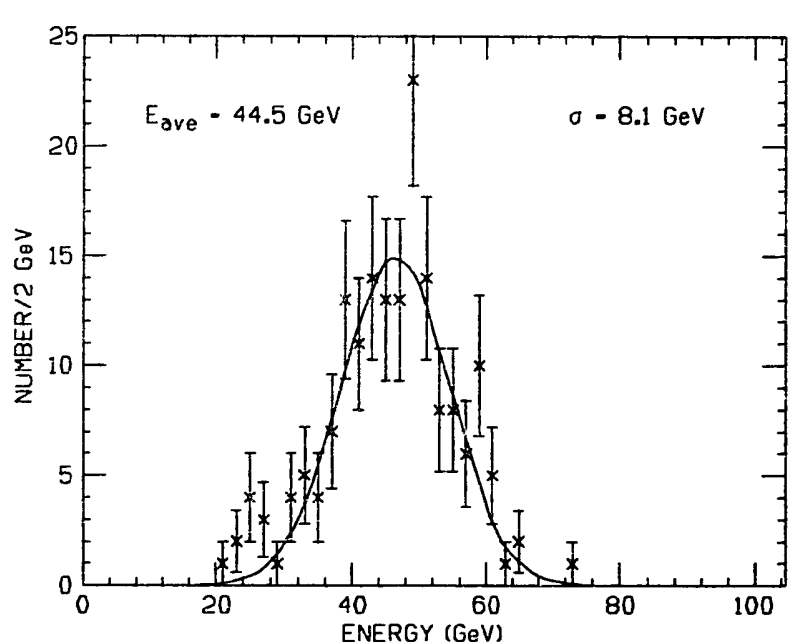

Fig. 8. Reconstructed shower energy in back-to-back SAM events for $115<\theta<160$ mrad.

muons, thereby providing an additional energy calibration check of the shower counter. We thus decided to sacrifice some resolution and correct, on the average, for saturation.

The saturation correction was derived from test beam running by comparing the response of individual wires to showers produced by one or two simultaneously incident $15 \mathrm{GeV}$ positrons. On average, i. ene energy deposited on a wire by two positrons is twice that of one positron. The amount by which the response of a wire deviates from this factor of two is a measure of

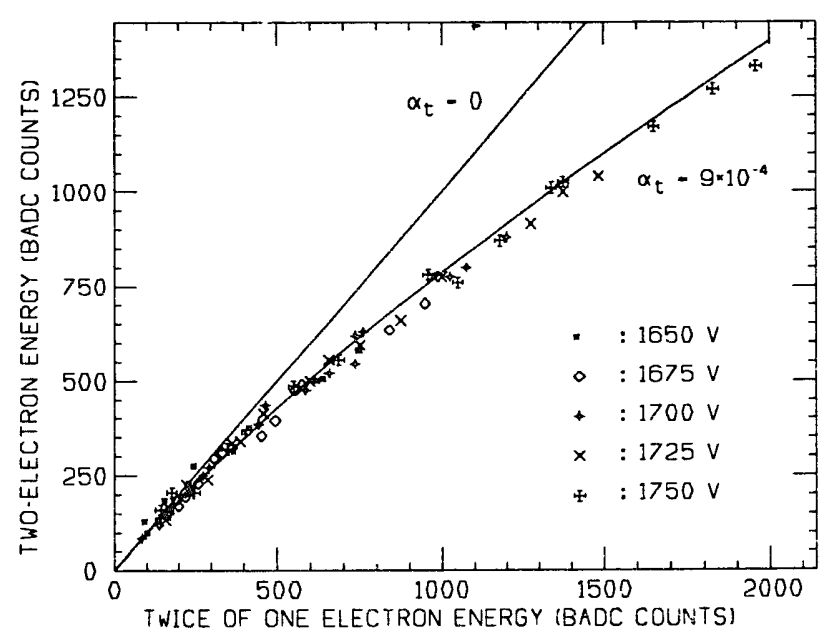

Fig. 9. For individual SAM calorimeter wires. the average response to two $15 \mathrm{GeV}$ positrons vs twice the one-positron response. The line labelled $\alpha_{1}=0$ is the shape expected if there were no saturation effect. The orher line shows the fitted saturation function $E_{\mathrm{c}}=E_{\mathrm{r}} \mathrm{e}^{\alpha_{\mathrm{t}} E_{\mathrm{r}}}$. the saturation. The data, shown in fig. 9 , were fitted assuming that

$E_{\mathrm{c}}=E_{\mathrm{r}} \mathrm{e}^{\alpha_{1} E_{\mathrm{r}}}$,

where $E_{\mathrm{r}}$ is the raw pulse height recorded by the ADCs, $E_{\mathrm{c}}$ is the corrected energy response, and $\alpha$ is a constant determined by the fit. This form has reasonable asymptotic properties, has only one parameter, and is partially motivated by the fact that wire response is exponential in voltage in the proportional region. It also describes the shape actually seen, as shown in fig. 9. The coefficient $\alpha_{t}$ depends on voltage and ADC scale.

Applying this function to the pulse height detected on each wire makes the summed response to multiple $15 \mathrm{GeV}$ positrons linear. It cannot recover the full resolution, however, for the following reason. The part of a shower seen by one wire may be concentrated on a small length of the wire, or may be spread out. The space charge effect will be much larger for the case where the shower energy is concentrated. Our saturation correction cannot take this variation into aciount. The saturation correction can be as large as a factor of 3 for wires with the largest pulse height.

To see the effect of the saturation correction, we have also reconstructed the events without the saturation corrections. The energy scale was reset using the new peak in the Bhabha distribution. Only 2 of the 839 events which formerly passed inclusive Bhabha cuts now failed, and no new events passed. We conclude that the systematic error due to saturation is negligible compared to statistical errors.

\section{Bhabha event selection}

\subsection{Overview}

Bhabha events were selected by requiring high energy showers $\left(>0.4 E_{\text {beam }}\right.$ ) in the fiducial area in both SAMs. Since the energy peak is at the beam energy, with only a small radiative tail, the energy cut is not critical. The fiducial position cuts are more sensitive because of the steeply falling $\theta^{-3}$ spectrum. The SAM tracking chambers were designed to give excellent $\theta$ resolution to minimize systematic errors associated with angular cuts, and have achieved the goal of $0.2 \mathrm{mrad}$ resolution. However, beam related backgrounds and extra tracking hits due to preshowering in the beam pipe have limited track reconstruction efficiencies to between $80 \%$ to $90 \%$. Calorimeter shower reconstruction, with an efficiency of $98 \%$, has thus been used for Bhabha event selection. The calorimeter resolution is abcut 1 mrad.

Possible systematic errors due to finite angular resolution and detector misalignments become negligible 
Table 2

Selection criteria for SAM Bhabha samples

\begin{tabular}{ll}
\hline Scheme & Cuts applied \\
\hline Inclusive & $E_{\mathrm{N}}, E_{\mathrm{S}}>0.4 E_{\text {beam }}$ \\
& $\theta_{\mathrm{N}}, \theta_{\mathrm{S}}<160 \mathrm{mrad}$ \\
Fiducial & $E_{\mathrm{N}}, E_{\mathrm{S}}>0.4 E_{\text {beam }}$ \\
& $\theta_{\mathrm{N}}, \theta_{\mathrm{S}}<160 \mathrm{mrad}$ \\
Precise (weight 1.0) & $\theta_{\mathrm{N}}>65 \mathrm{mrad}, \theta_{\mathrm{S}}>65 \mathrm{mrad}$ \\
or & or \\
Gross (weight 0.5) & $\hat{\theta}_{i}>65 \mathrm{mrad}, 60<\theta_{j}<65 \mathrm{mrad}$ \\
\hline
\end{tabular}

by the use of a "Gross-Precise" scheme [6], which employs a larger angular fiducial region on one end of the detector than on the other. While it is necessary to limit the fiducial area used in the Gross-Precise scheme to the more central region of the SAM (in order to have an accurately calculable absolute cross section), an "Inclusive" sample which includes events closer to the edges of the detector can also be used for determining luminosities at different energy scan points. The cuts defining these two samples are summarized in table 2 . In all cases, non-Bhabha background is negligible.

\subsection{Cross section detected with Gross-Precise calorime- ter scheme}

The Gross-Precise scheme of fiducial cuts minimizes the systematic errors by using only the well understood regions of the SAM, and using a weighting scheme to cancel many possible systematic errors. It is relatively straightforward, and very robust.

We used the reconstructed positions from the calorimeter for the fiducial cuts. The tracking chambers provided an independent measure of the calorimeter resolution and of the effects of inner-edge leakage, described below. (A slightly more complicated analysis using the tracking chambers for defining cuts yields results consistent with those presented here [5].)

One standard deviation in lateral shower energy profile corresponds to about two cells, or slightly more than $10 \mathrm{mrad}$ in $\theta$. Intuitively, one thus expects that position and energy are well measured for showers more than 10 mrad from the edge of the device. Fig. 10 illustrates the expected effect using EGS. We chose 60 mrad as the innermost usable edge of SAM, an angle well away from the SAM inner cdge and from any possible mask obstruction.

The Gross-Precise scheme can be thought of as using a single arm spectrometer to measure Bhabhas. One picks a "Precise" fiducial area on one end which sets the cross section, and simply counts showers into this area. To reduce possible backgrounds, one tags these events as Bhabha events by requiring a shower in a larger "Gross" fiducial area on the other end, large enough to allow for resolution effects and for misalignment of the IP with respect to the detector. An event which has showers in the Precise fiducial area of both ends can be thought of as being in two single-arm spectrometers. It is assigned twice the weight of an event with a shower in the Precise fiducial area of just one end. With this weighting scheme, changes in the

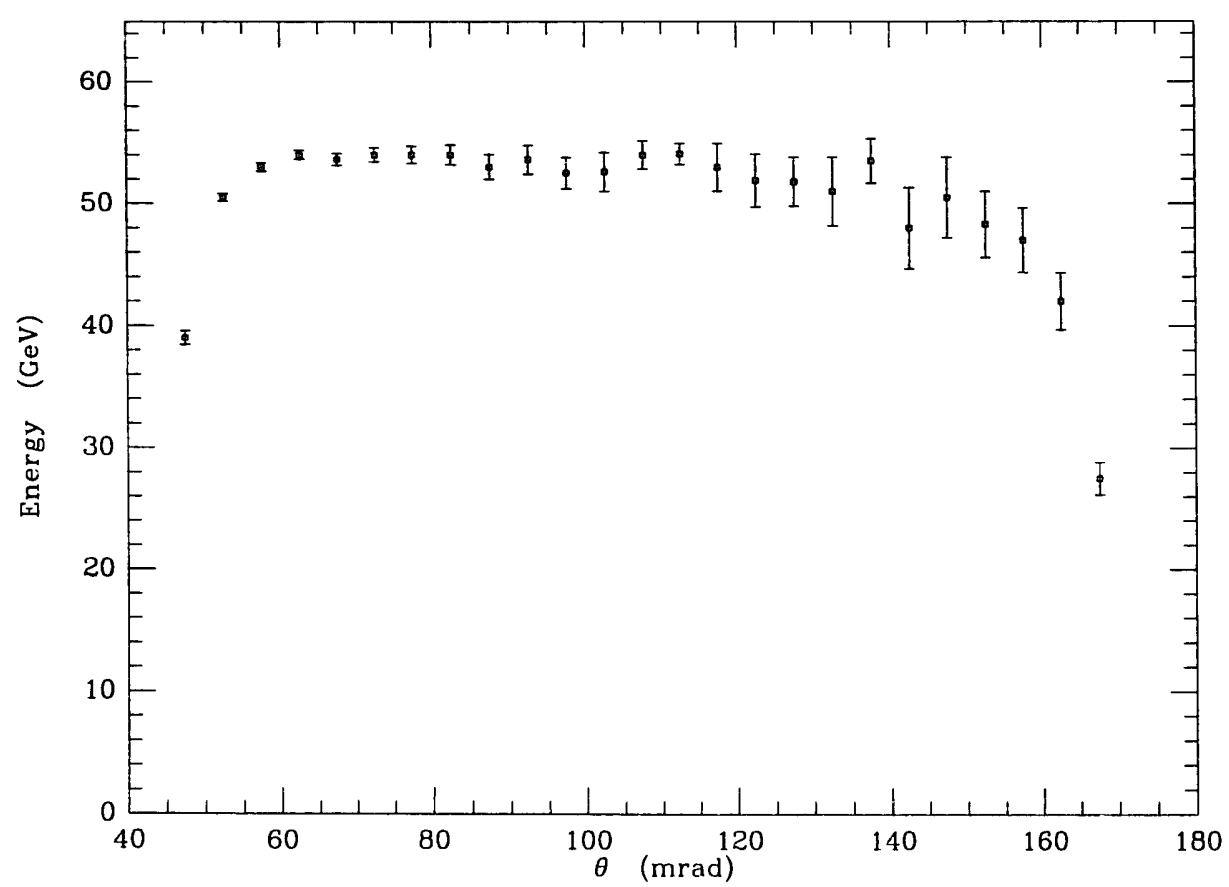

Fig. 10. Reconstructed shower energy versus $\theta$ from an EGS Monte Carlo simulation. 
accepted cross section due to detector offsets, rotations, and resolution effects are canceled to first order.

The difference between Gross and Precise apertures of $5 \mathrm{mrad}$ selected in this analysis allows for roughly $3 \mathrm{~mm}$ of IP offset in $X$ or $Y, 60 \mathrm{~mm}$ of IP offset in $Z$, or $8 \mathrm{~mm}$ detector position uncertainty. The IP position in $X$ and $Y$ has been located to better than $1 \mathrm{~mm}$ (see figs. 3 and 4), the IP offset in $Z$ is less than $15 \mathrm{~mm}$, and the detector resolution is $2 \mathrm{~mm}$, all measured using the SAM itself with detected Bhabhas. The Gross aperture is thus adequate.

The angle of the beam axis with respect to the SAM axis has been determined by a fit to the azimuthal distribution of Precise events. It is consistent with zero, but has a 3 mrad statistical error. A 3 mrad rotation of the SAM would correspond to a $0.4 \%$ change in the cross section. This alignment has been cross checked by observing Bhabha events in the mini-SAM detector [1], a set of shower counters at smaller angles to the beam axis than the SAM detector. The difference in alignment between the SAM and mini-SAM detectors was found to be less than $1.5 \mathrm{mrad}$. The effect on measured luminosities due to any remaining uncertainties in aligment is negligible compared to statistical errors.

\subsection{Correction for $\theta$ skewing}

Although symmetric position-resolution smearing cancels to first order in the Gross-Precise scheme, resolution effects that are not symmetric can have an effect. Leakage of shower energy from the inner edge systematically shifts measured shower positions to larger $\theta$. We measured this effect by comparing positions obtained with the tracking chambers to those from the calorimeters. Fig. 11 shows a plot of this difference for showers in the range $60<\theta<70 \mathrm{mrad}$, with a Gaussian fit superimposed. The fit indicates a $0.44 \pm 0.10 \mathrm{mrad}$ systematic shift due to this effect. Fig. 12 shows the shift as a function of $\theta$ for both data and EGS simulations. The data show somewhat larger shifts near the edge than the Monte Carlo. We correct the cross section by $+1.6 \%$, corresponding to the shift of

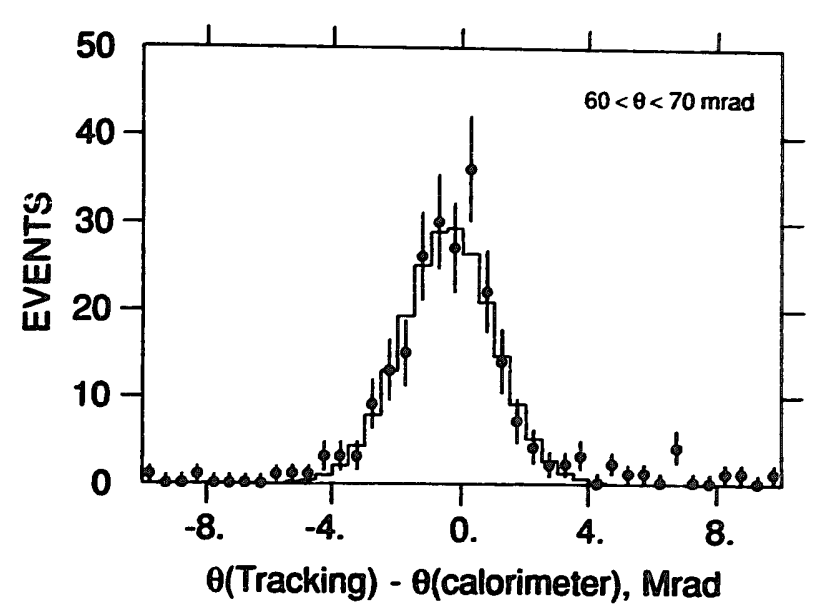

Fig. 11. $\theta_{\text {tracking }}-\theta_{\text {calorimeter }}$ for showers with $60<\theta<70$ mrad, which is the region where the fiducial cut is made. The Gaussian fit (histogram) shows a $\mathbf{- 0 . 4}$ mrad average skewing of the shower reconstruction due to energy leakage at the inner edge of the SAM. (See table 3.)

$0.5 \mathrm{mrad}$ seen in the data, but assign a conservative $\pm 1.6 \%$ systematic error to this correction.

\subsection{Reconstruction efficiency}

The data corresponding to the first 250 Bhabha events has been scanned by eye. Comparing to the full reconstruction, one evidently good event in the scanned sample was found which was not selected by the reconstruction algorithm. The electron in this event passed through approximately three radiation lengths of material before hitting the SAM, making it more difficult to reconstruct.

In parallel, we have used another line of event scanning which requires only one reconstructed shower in the SAMs. All such events have been scanned. The scan indicates that the reconstruction efficiency is (98.1 $\pm 1.2) \%$. The inefficiency is concentrated in the "preshower" region described in section 4 .

Table 3

Corrections to and estimated systematic errors of the SAM Bhabha cross section at $E_{\mathrm{cm}}=91.1 \mathrm{GeV}$

\begin{tabular}{lccc}
\hline & Correction & Estimated error & Cross section \\
\hline$\sigma_{\mathrm{GP}}$ (M.C. stat) & & & $25.3 \mathrm{nb} \pm 0.4 \%$ \\
Inner edge energy leakage & $+1.6 \%$ & $\pm 1.6 \%$ & \\
120-160 mrad recon. ineff. & $-1.9 \%$ & $\pm 1.2 \%$ & \\
$\quad$ Higher order rad. corr. & $\pm 2.0 \%$ & & $25.2 \mathrm{nb} \pm 2.8 \%$ \\
$\begin{array}{l}\sigma_{\mathrm{GP}} \text { corrected } \\
\text { Ave. scale for Inclusive events }\end{array}$ & 1.675 & $\pm 2.8 \%$ & $42.2 \mathrm{nb} \pm 4.0 \%$ \\
$\begin{array}{l}\sigma_{\text {Inclusive scaled, ave. }}^{\text {(scan points 1-7) }} \\
\text { (scan points 8-10) }\end{array}$ & & $(42.4 \mathrm{nb})$ \\
\hline
\end{tabular}




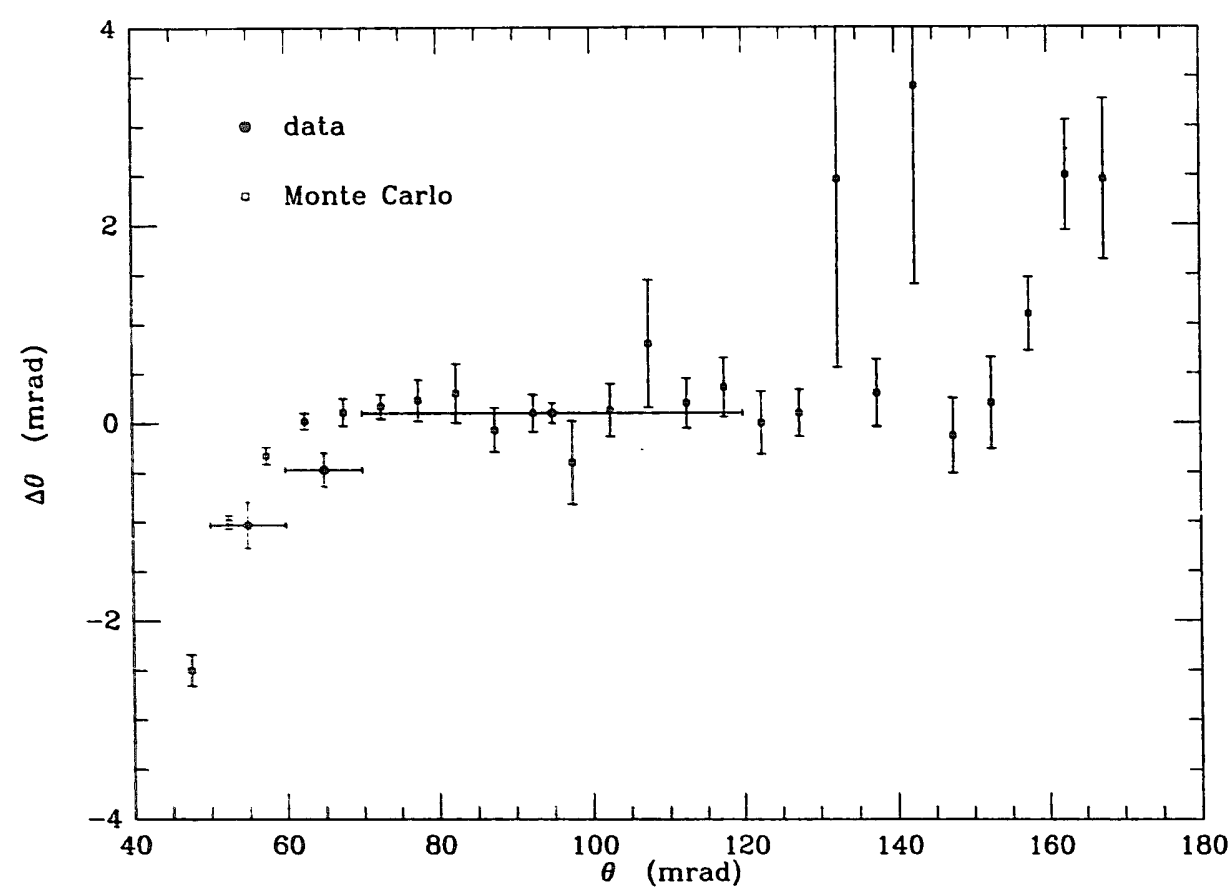

Fig. 12. Difference in $\theta$ between tracking chamber and calorimeter reconstruction as a function of $\theta$ for both data and Monte Carlo.

\subsection{Scaled inclusive cross section}

Assuming that SAM-to-mask-to-beam alignment does not change during the running, the subsample of Inclusive Bhabha events which failed to pass the fiducial area cuts can still be used to help measure the relative point-to-point luminosity in the energy scan over the $Z^{0}$ resonance. This improves the measurement of the mass and width, but not of the height of the resonance. A convenient way to put this information into the fit is to derive a cross section corresponding to the selection of Inclusive events. This is obtained by scaling the fiducial cross section by the ratio of Inclusive to Gross-Precise events. One obtains a reduced statistical error in the resonance fitting by using the larger sample of Inclusive events, but at the cost of an overall "systematic" (actually statistical) error due to the scaling of the cross section.

Assuming no change in alignments, the cross section scale factor would be just

$\left(\frac{N_{\text {Inclusive }}}{N_{\mathrm{GP}}}\right)=\frac{839}{501}=1.675 \pm 0.047$

where binomial statistics are used to calculate the error since Gross-Precise events are a subset of Inclusive events. However, the beam-pipe was realigned at run 19265 , at the end of scan point number 7. Based on surveys before and after the realignment, this changed the Inclusive cross section by $(-1 \pm 2) \%$. Additionally, the beam orbit was changed at times by up to $600 \mu \mathrm{m}$, but the effect on the detected cross section is less than $1 \%$. The average resulting scaled Inclusive cross section is $\sigma_{\text {Inclusive }}=42.2 \pm 1.2$ (stat) $\mathrm{nb}$ at $91.1 \mathrm{GeV}$. As a cross check, we calculated $\sigma_{\text {Inclusive }}$ using beam mask positions and an EGS model of inner edge leakage. The result of $44.3 \pm 1.8$ (sys) nb, with the error coming from alignment and shower profile uncertainties, is consistent with that derived above but has more poorly understood systematics.

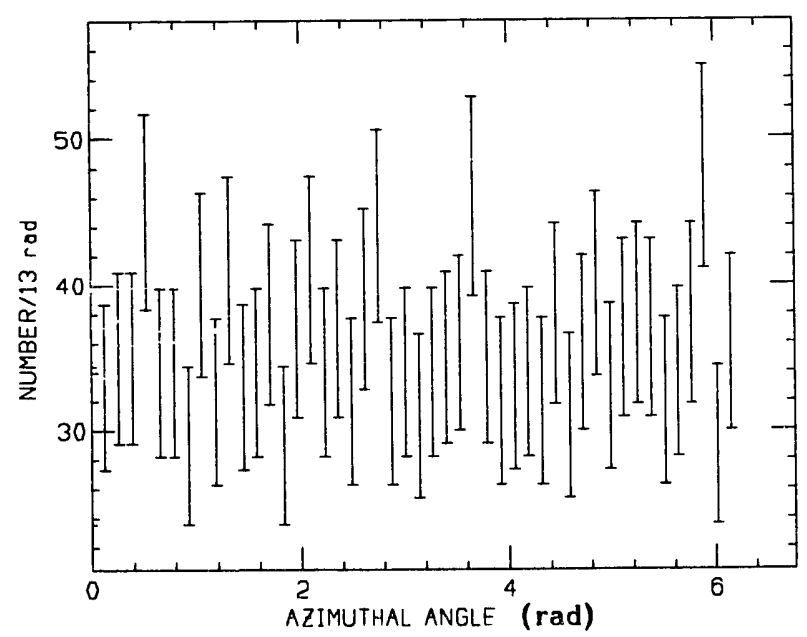

Fig. 13. Azimuthal angle distribution of reconstructed showers in the SAM, for events which pass Inclusive fiducial cuts. 

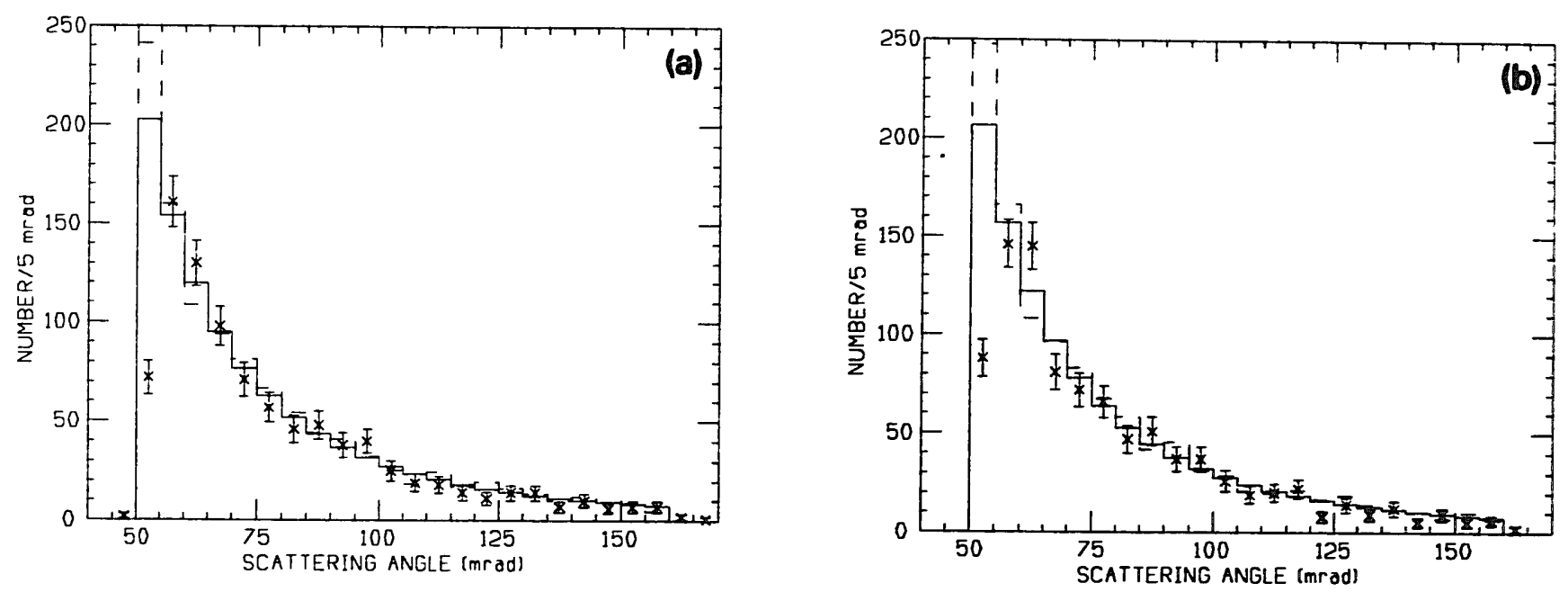

Fig. 14. (a) Distribution in $\theta$ of reconstructed showers in the north SAM, for Bhabha events that pass Inclusive fiducial cuts. Solid histogram is $\theta^{-3}$, as expected from lowest order QED. Dashed histogram shows Bhabha Monte Carlo with EGS showers, normalized to the distribution in the angular range $60<\theta<115 \mathrm{mrad}$. (b) Same as (a) but for south SAM.

\section{Bhabha physics distributions}

Fig. 13 shows that events in the SAM are isotropic in azimuth, as expected for Bhabhas. Fig. 14 indicates that the angular distribution is consistent with a $\theta^{-3}$ behavior. The measured acollinearity distribution shown in fig. 15 is also in agreement with expectation. Non-Bhabha backgrounds would show up very clearly in this plot at large values of acollinearity.

\section{Monte Carlos and radiative corrections}

Table 4 gives a comparison of the accepted Bhabha cross section, without detector resolution corrections,

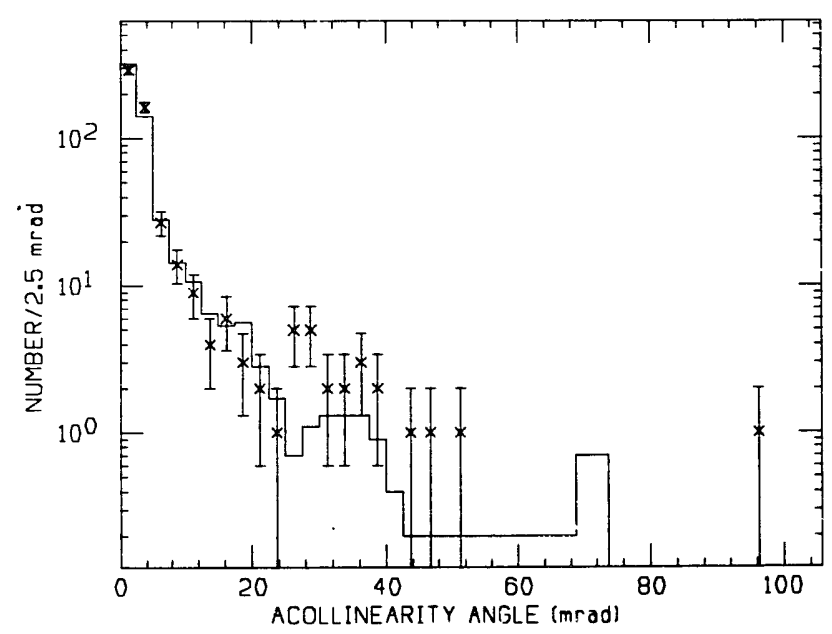

Fig. 15. Acollinearity distribution for Bhabha candidates passing Inclusive cuts. Histogram is from a Bhabha Monte Carlo with EGS showers. derived from various Monte Carlo generators. While no complete Bhabha Monte Carlo generator exists for the entire angular range at $Z^{0}$ energies, in that complete first order weak corrections and the corrections beyond the first order for the initial state photon radiation are not both implemented, the agreement between different generators is good, differing typically by a percent or less. From the above comparison, and from a previous experimental comparison of small angle Bhabha scattering [7], we are confident that the effect of higher-order corrections is less than $2 \%$.

Table 4

Comparison of Bhabha Monte Carlo generators. The cross sections contain corrections of 0.1 to $0.4 \mathrm{nb}$ for $\mathrm{e}^{+} \mathrm{e}^{-} \boldsymbol{\gamma}$ events where one $e$ is nearly collinear with the beam axis. Errors quoted are statistical only

\begin{tabular}{|c|c|c|}
\hline Generator & $\sigma_{\mathrm{SAM}}[\mathrm{nb}]$ & Comments \\
\hline EEG [9] & $25.3 \pm 0.1$ & $\begin{array}{l}\mathrm{e}^{+} \mathrm{e}^{-} \text {and } \mathrm{e}^{+} \mathrm{e}^{-} \gamma \text { from first- } \\
\text { order electromagnetic and } Z^{0}- \\
\text { photon interference }\end{array}$ \\
\hline EEG-X & $25.2 \pm 0.1$ & $\begin{array}{l}\text { EEG with exponentiation of } \\
\text { hard and/or soft part to } \\
\text { estimate higher-order radiative } \\
\text { corrections }\end{array}$ \\
\hline BHK [10] & $25.4 \pm 0.1$ & $\begin{array}{l}\text { also known as } \mathrm{BABMC} \text { and } \\
\text { BHABHAMC, } \mathrm{e}^{+} \mathrm{e}^{-} \text {and } \\
\mathrm{e}^{+} \mathrm{e}^{-} \gamma \text { electroweak consistently } \\
\text { to first order }\end{array}$ \\
\hline BHLUMI [11] & $25.1 \pm 0.1$ & $\begin{array}{l}\mathrm{e}^{+} \mathrm{e}^{-} \text {plus any number of } \\
\text { photons, uses YFS exponentia- } \\
\text { tion for higher-order electro- } \\
\text { magnetic corrections; includes } \\
\mathrm{Z}^{0} \text {-photon interference }\end{array}$ \\
\hline
\end{tabular}


Table 5

Bhabhas detected in SAM and the resulting calculated luminosity, listed by beam energy scan point. Errors listed are statistical only. Total $\int L \mathrm{~d} t=19.97 \pm 0.69$ (stat $) \pm 0.80(\mathrm{sys}) \mathrm{nb}^{-1}=19.97 \pm 1.06 \mathrm{nb}^{-1} . \sigma_{M}(\gamma)$ scales as $E_{\mathrm{cm}}^{-2}$ over the scan region. $\sigma_{M}(\gamma \mathrm{Z})$, the $Z^{0}$-photon interference term, is giv. $\mathrm{n}$ for $M_{\mathrm{Z}}=91.14 \mathrm{CeV} . \sigma_{\text {Inclusive }}$ is the total Inclusive cross section for each energy, using the "Inclusive scaled to Gross-Precise" scheme

\begin{tabular}{clllllllr}
\hline $\begin{array}{l}\text { Scan } \\
\text { point }\end{array}$ & $\begin{array}{l}E_{\mathrm{cm}} \\
{[\mathrm{GeV}]}\end{array}$ & $\begin{array}{l}\sigma_{M}(\gamma) \\
{[\mathrm{nb}]}\end{array}$ & $\begin{array}{l}\sigma_{M}(\gamma \mathrm{Z}) \\
{[\mathrm{nb}]}\end{array}$ & $\begin{array}{l}\sigma_{\text {Inclusive }} \\
{[\mathrm{nb}]}\end{array}$ & $N_{\text {Inclusive }}$ & $N_{\text {Precise }}$ & $N_{\text {Gross }}$ & $\begin{array}{c}L \mathrm{~d} t \\
{[\mathrm{nb}}\end{array}$ \\
\hline 1 & 92.16 & 41.45 & -0.47 & 40.98 & 33 & 19 & 1 & $0.80 \pm 0.14$ \\
2 & 90.74 & 42.75 & 0.30 & 43.05 & 54 & 34 & 7 & $1.25 \pm 0.17$ \\
3 & 89.24 & 44.20 & 0.44 & 44.64 & 24 & 14 & 0 & $0.54 \pm 0.11$ \\
4 & 91.50 & 42.04 & -0.26 & 41.78 & 53 & 30 & 4 & $1.27 \pm 0.17$ \\
5 & 89.98 & 43.48 & 0.48 & 43.96 & 36 & 23 & 1 & $0.82 \pm 0.14$ \\
6 & 92.96 & 40.73 & -0.44 & 40.29 & 43 & 23 & 3 & $1.07 \pm 0.16$ \\
7 & 91.06 & 42.45 & 0.06 & 42.51 & 171 & 93 & 5 & $4.02 \pm 0.31$ \\
8 & 91.43 & 41.68 & -0.22 & 41.47 & 165 & 90 & 7 & $3.98 \pm 0.31$ \\
9 & 92.22 & 40.98 & -0.48 & 40.50 & 128 & 78 & 5 & $3.16 \pm 0.28$ \\
10 & 90.35 & 42.70 & 0.44 & 43.14 & 132 & 78 & 5 & $3.06 \pm 0.27$ \\
Total & & & & & 839 & 482 & 38 & $19.97 \pm 0.69$ \\
\hline
\end{tabular}

\section{Luminosity calculation}

Finally, table 5 shows a summary of the scan points and the luminosity calculated from SAM events for each point [8]. The table shows luminosities based on the "Inclusive scaled to Gross-Precise" scheme, and yields total $\int L \mathrm{~d} t=19.97 \pm 0.69$ (stat) \pm 0.80 (sys) $\mathrm{nb}^{-1}$. If one uses the Gross-Precise scheme alone, one obtains total $\int L \mathrm{~d} t=19.93 \pm 0.89$ (stat) \pm 0.56 (sys) $\mathrm{nb}^{-1}$. This has the same total error, as it must, since the Inclusive scheme only uses the additional Inclusive events to obtain a better relative luminosity between the scan points.

\section{Conclusion}

A precision luminosity measurement has been performed at the SLC by measuring Bhabha scattering in the angular region from 60 to $160 \mathrm{mrad}$ with detectors consisting of nine layers of drift tubes followed by six layers each containing lead and proportional tubes. These small-angle monitors (SAMs) surrounded both outgoing beams and achieved a Bhabha angular resolution of $0.2 \mathrm{mrad}$ using the tracking layers, and approximately $1.0 \mathrm{mrad}$ with the calorimeter alone. The energy resolution was measured to be $(14 \pm 2) \%$ at 46 $\mathrm{GeV}$. The trigger for Bhabha events demanded either a deposit in excess of $4 \mathrm{GeV}$ in each of the two SAMs or a deposit of at least $7 \mathrm{GeV}$ in one of the SAMs. The reconstruction of the calorimetric data included corrections for saturations and shower leakage. The overall event reconstruction efficiency was estimated to be $(98.1 \pm 1.2) \%$. Bhabha cross sections were determined using a number of different event selection criteria and several independent Bhabha Monte Carlo generators for obtaining an absolute normalization. The total inte- grated luminosity measured at ten energies near the $\mathbf{Z}$ boson resonance during the April-October 1989 run is $19.97 \pm 0.69$ (stat) \pm 0.80 (sys) $\mathrm{nb}^{-1}$, corresponding to a total of 839 detected Bhabha events.

\section{References}

[1] G.S. Abrams et al., Nucl. Instr. and Meth. A281 (1989) 55.

[2] E.L. Cisneros, H.K. Kang, J.N. Hall and R.S. Larsen, IEEE Trans. Nucl. Sci. NS-24 (1977) 413.

[3] M. Breidenbach, E. Frank and D. Nelson, IEEE Trans. Nucl. Sci. NS-25 (1978) 706.

[4] The EGS Code System (Version 3), R.L. Ford and W.R. Nelson, SLAC-210, (1978) is still used in the Mark II HOWL Monte Carlo. The EGS4 Code System, W.R. Nelson, H. Hirayama and D.W.O. Rogers, SLAC-265, (1985), has also been used for some of the SAM studies.

[5] S.J. Hong, Ph.D. Thesis, University of Michigan (1990).

[6] G. Barbiellini et al., Nucl. Instr. and Meth. 123 (1975) 125

J.F. Crawford et al., Nucl. Instr. and Meth. 127 (1975) 173 ;

L.H. O'Neill et al., Phys. Rev. Lett. 37 (1976) 395;

U. Amaldi et al., Nucl. Phys. B145 (1978) 367; and

Brian Keith Heltsley, Ph.D. Thesis, WISC-EX-83/233, 1983.

[7] C. von Zanthier et al., Phys. Rev. D 43 (1991) 34.

[8] Some of the numbers in table 5 differ slightly from values published previously in G.S. Abrams et al., Phys. Rev. Lett. 63 (1989) 2173 primarily because the previous values used both SAM and mini-SAM detectors, while the present one uses only SAM. Also, all of the data for scan point 10 is included here.

[9] F.A. Berends and R. Kleiss, Nucl. Phys. B228 (1983) 537.

[10] F. Berends, R. Kleiss, W. Hollik, Nucl. Phys. B304 (1988) 712.

[11] S. Jadach, B.F.L. Ward, Phys. Rev. D 40 (1989) 3582. 